\title{
On the activation of $\mathrm{PhICl}_{2}$ with pyridine
}

Tiffany B. Poynder, ${ }^{a}$ Analia I. Chamorro Orué, ${ }^{\mathrm{b}}$ Tania, ${ }^{\mathrm{a}}$ Lachlan Sharp-Bucknall,, ${ }^{\mathrm{a}}$ Matthew T. Flynn, ${ }^{a}$ David J. D. Wilson, ${ }^{a}$ Kasun S. Athukorala Arachchige, ${ }^{b}$ Jack K. Clegg ${ }^{b *}$ and Jason L. Dutton $^{\text {a* }}$

aDepartment of Chemistry and Physics, La Trobe University, Melbourne, Victoria, Australia

${ }^{b}$ School of Chemistry and Molecular Biosciences, The University of Queensland, St Lucia, Queensland 4072,

Email: j.clegg@uq.edu.au, j.dutton@latrobe.edu.au

\section{ABSTRACT}

It has been previously proposed that pyridines can activate $\mathrm{PhICl}_{2}$ by displacing a chloride and forming the $[\mathrm{Phl}(\mathrm{Pyr})(\mathrm{Cl})]^{+}$cation as a reactive intermediate. Here we show that pyridine does not displace chloride, but rather forms a weak complex with the iodine via halogen bonding along the $\mathrm{C}-\mathrm{I}$ bond axis. This interaction is investigated by NMR, structural, charge density and theoretical investigations, which all indicate the pyridine does not activate $\mathrm{PhICl}_{2}$ as proposed.

Keywords: lodine, hypervalent, oxidants, halogen bonding, charge density 


\section{INTRODUCTION}

$\mathrm{PhICl}_{2}$ is a versatile oxidant, primarily acting as a chlorinating agent representing a convenient substitute for $\mathrm{Cl}_{2} . \mathrm{Cl}_{2}$ is a highly corrosive, toxic gas, which in addition to being hazardous, is challenging to deliver in a stoichiometric fashion. $\mathrm{PhICl}_{2}$ conversely is an easily weighed solid and has been used widely in the oxidation of organic and inorganic compounds. ${ }^{1-3}$

In a series of recent papers Murphy and co-workers have reported on the activation of $\mathrm{PhICl}_{2}$ using catalytic amounts of pyridine ligands to achieve chlorination of diazo compounds or hydrozones (Scheme 1). ${ }^{4-8}$

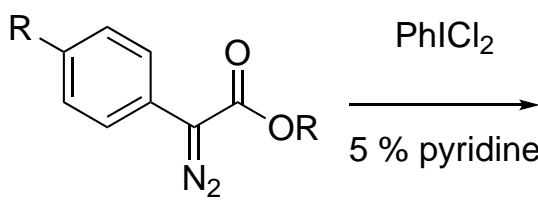<smiles>[R]c1ccc(C(Cl)(Cl)C(=O)OCc2ccccc2)cc1</smiles><smiles>CC(C)N1C(=O)C(=N)c2ccccc21</smiles>

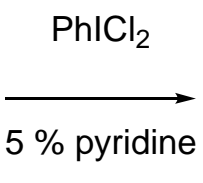<smiles>CC(C)N1C(=O)C(Cl)(Cl)c2ccccc21</smiles><smiles>O=C1/C(=N\NSc2ccccc2)c2ccccc2N1[O-]</smiles>

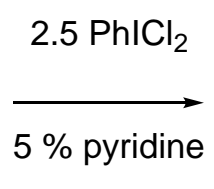<smiles>CC(C)N1C(=O)C(Cl)(Cl)c2ccccc21</smiles>

Scheme 1. Reported reactions of $\mathrm{PhICl}_{2}$ with diazo or hydrozone compounds using catalytic pyridine.

The mechanism presented for the initial report regarding the chlorination of diazo compounds proposed that pyridine displaces a chloride from $\mathrm{PhICl}_{2}$ giving activated cationic complex $\mathbf{1}$, which is then susceptible to attack by the nucleophilic carbon of the diazo (Scheme 2). ${ }^{4}$ 


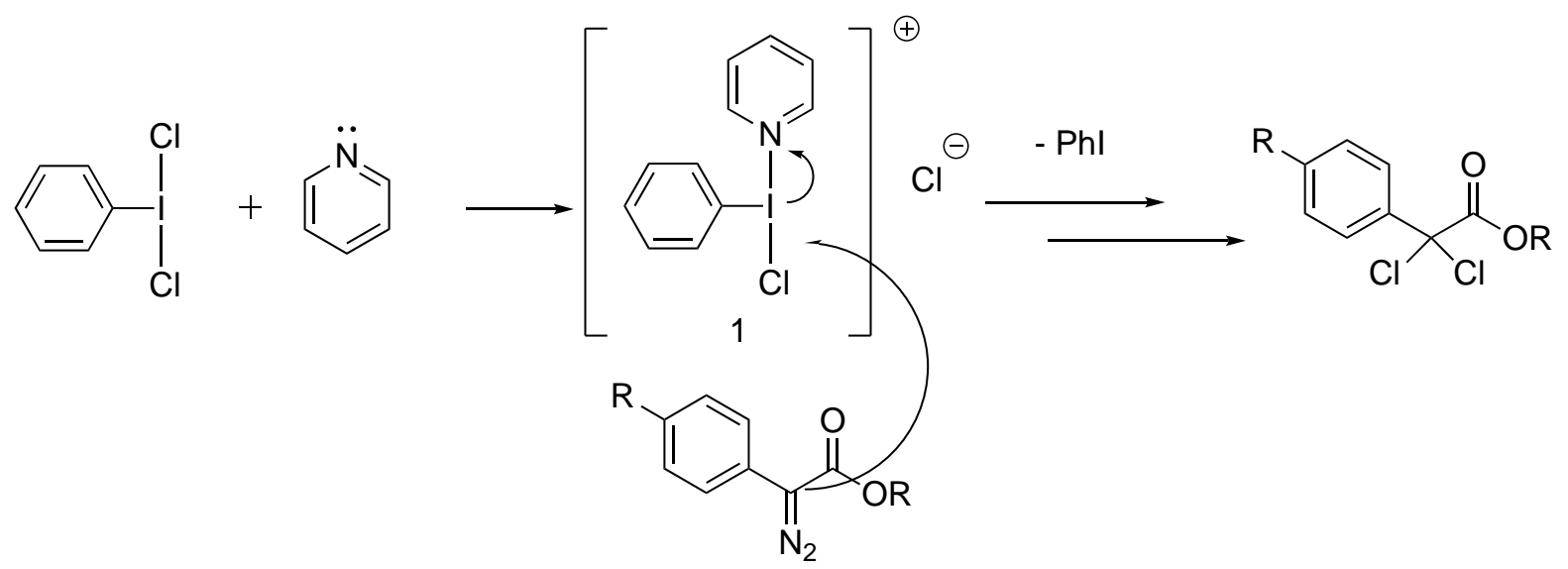

Scheme 2. Proposed activation of $\mathrm{PhICl}_{2}$ with pyridine.

Evidence for activated complex 1 was provided by a downfield shift in the ${ }^{1} \mathrm{H}$ NMR resonance of the methyl protons in the proposed adduct with lutidine of $\sim 0.5 \mathrm{ppm} .{ }^{4}$ 4-DMAP is also used as an activating pyridine ligand in the reports. ${ }^{5}$ As part of our interest in the coordination chemistry of I(III) with $\mathrm{N}$-ligands the proposed active species 1 caught our attention as we have been attempting unsuccessfully to isolated such a complex for some time. In this report we describe our findings on the interaction of $\mathrm{PhICl}_{2}$ with pyridine ligands.

\section{RESULTS AND DISCUSSION}

$\mathrm{PhICl}_{2}$ was prepared by oxidation of iodobenzene with $\mathrm{H}_{2} \mathrm{O}_{2}$ in the presence of concentrated $\mathrm{HCl}$ (ESI) and crystallised from a saturated $\mathrm{CH}_{2} \mathrm{Cl}_{2}$ solution $\left(-20^{\circ} \mathrm{C}\right)$. While the crystal structure of $\mathrm{PhICl}_{2}$ has been previously reported, ${ }^{9}$ the high quality of the crystals obtained allowed the collection of diffraction data to better than $0.38 \AA$ resolution and refinement of a multipole model (Figure 1). ${ }^{10}$ In the solid state the iodine atom has the expected T-shaped geometry with a slight asymmetry due to the presence of different intermolecular interactions involving each $\mathrm{Cl}$ atom (see ESI). The full deformation of the static electron density (Figure 2) and 2D-Lapacian maps (Fig. S3-S5, ESI) indicates a local partial positive charge ( $\sigma$-hole) on the iodine atom opposite the phenyl group. ${ }^{11}$ The $\sigma$-hole acts as a halogen bond acceptor from an adjacent chlorine $\left(\mathrm{Cl} 2 \cdots \mid 1=3.42 \AA, R_{\mathrm{ClI}}=0.92, \psi=0.31\right) .{ }^{12}$, 
${ }^{13}$ The presence of this type $\beta$ halogen-bond was confirmed by a $(3,-1)$ bond critical point (Fig. S6, ESI) with a density of $\rho=0.16 \mathrm{e} \cdot \AA^{-3}$ (Table S5, ESI).

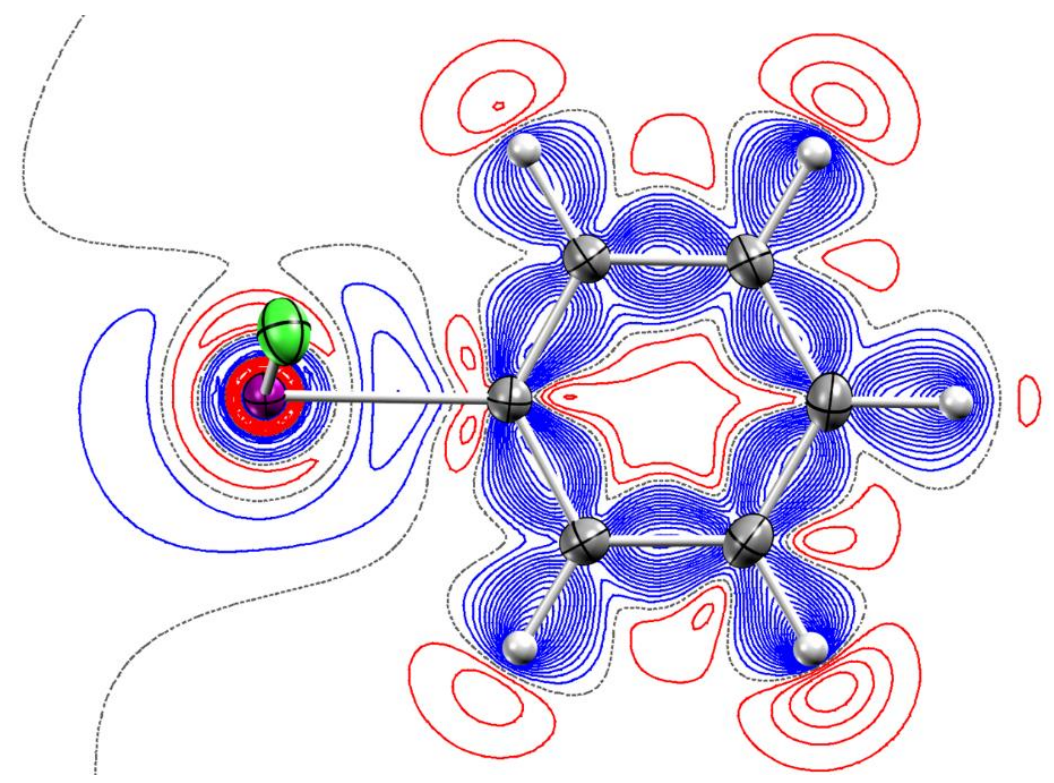

Figure 1. ORTEP representation (90\% probability level) and 2D-deformation electron density of compound $\mathrm{PhICl}_{2}$ viewed perpendicular to the ZX plane of the phenyl ring (contour $0.05 \mathrm{e}^{-1}$ ). Positive density is shown in blue and negative in red; grey dotted lines represent zero density. $11-C 1=2.0977(4)$

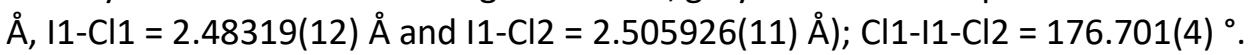


The reaction of $\mathrm{PhICl}_{2}$ with pyridine was then investigated. A sample of $\mathrm{PhICl}_{2}$ was dissolved in $\mathrm{CDCl}_{3}$. One stochiometric equivalent of pyridine was added and an NMR spectrum was taken. The ${ }^{1} \mathrm{H}$ NMR spectrum showed no significant change in the chemical shift for either the pyridine or $\mathrm{PhICl}_{2}$ moieties, but a slight broadening was observed as compared to the separate species under the same conditions. We also reproduced the work with 1 equivalent of lutidene from Murphy showing the same slight change in chemical shift as they reported. ${ }^{4}$ This is in contrast to a related pyridine complex of I(III), Weiss' reagent, $\left[\mathrm{PhI}(\mathrm{Py})_{2}\right]^{2+}$, in which pyridine is unambiguously bound, where the ortho pyridine $\mathrm{C}-\mathrm{H}$ protons are shifted to 9.00 ppm, as compared to $8.57 \mathrm{ppm}$ for free pyridine. ${ }^{14}$ The sample was placed in the freezer at 30 deg $C$ and pale yellow needle-like crystals formed overnight. Single crystal X-ray analysis returned a structure of only $\mathrm{PhICl}_{2}$.
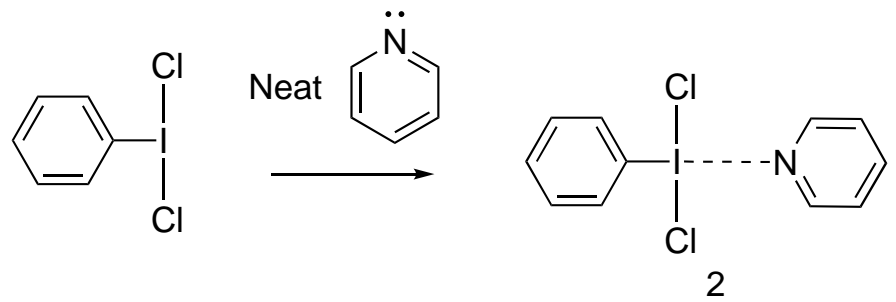

Scheme 3. Reaction of $\mathrm{PhICl}_{2}$ with neat pyridine.

A sample of $\mathrm{PhICl}_{2}$ was then dissolved in neat pyridine and held at $-30{ }^{\circ} \mathrm{C}$ overnight which resulted in the precipitation of bright yellow block-like crystals. Single crystal X-ray analysis (Figure 2) confirmed the formation of a $\mathrm{PhICl}_{2} \cdot \mathrm{Py}$ complex (2). 


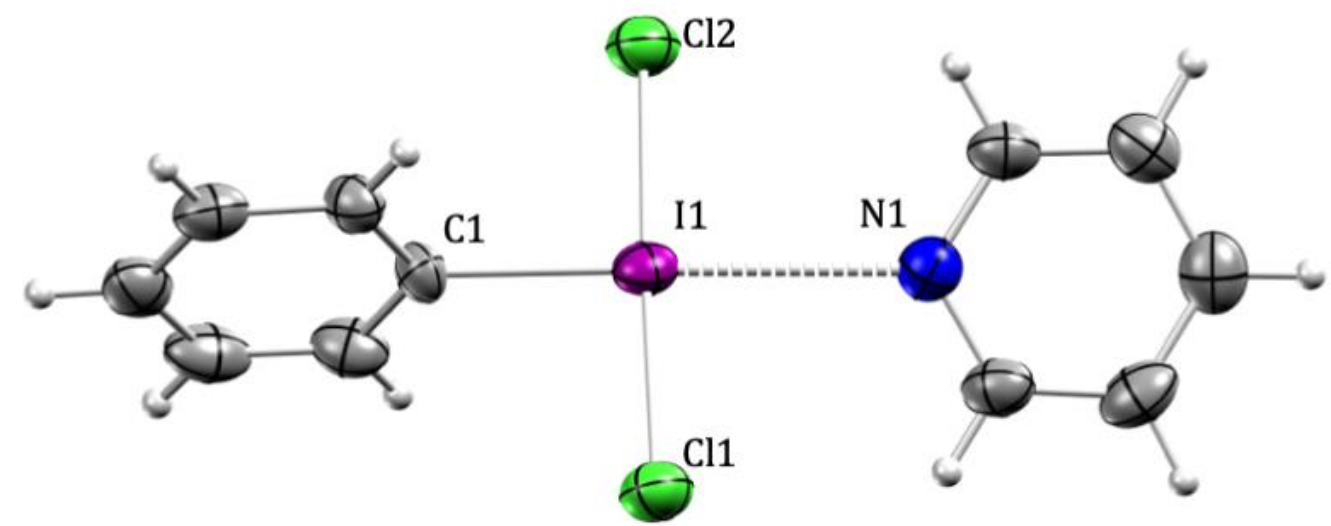

Figure 2. ORTEP representation of the crystal structure of the $\mathrm{PhICl}_{2} \cdot \mathrm{Py}$ complex (2) shown with $70 \%$

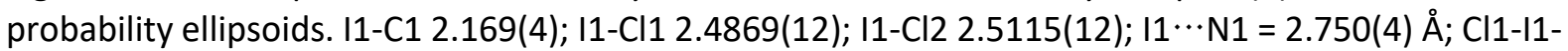
$\mathrm{Cl} 2=178.05(4)^{\circ}$.

The $\mathrm{PhICl}_{2}$ part of the molecule in the structure of $\mathbf{2}$ is very similar to the structure of $\mathrm{PhICl}_{2}$ with a slightly elongated C-I bond (2.169(4) A in 2 compared to $2.0977(4) \AA$ in 1 ). The pyridine is located opposite the phenyl ring resulting in a square planar iodine geometry. The $\mathrm{N}-\mathrm{I}$ distance is 2.750 (4) $\AA$ which is indicative of the presence of a much stronger type $\alpha$ halogen bond than in $1\left(R_{\mathrm{NI}}=0.78, \psi=0.98\right),{ }^{12,}{ }^{13}$ but significant longer than those present in Weiss' reagent $(2.22 \AA), 14,15$ bipyridine adduct of $\left[\mathrm{ICl}_{2}\right]^{+}(2.27 \AA),{ }^{16}$ or the 4 -coordinate pyridine adduct of iodine triacetate $(2.406 \AA) .{ }^{17}$ NMR titration experiments followed by fitting using BindFit ${ }^{18,19}$ with $1,2,3,4,5$ and 10 equivalents of pyridine relative to $\mathrm{PhICl}_{2}$ in $\mathrm{CDCl}_{3}$ gave a binding constant of $0.7 \mathrm{M}^{-1}$ and binding behavior consistent with a host-guest or coordinative interaction, rather than displacement. Taking together these observations indicate pyridine does not displace a chloride from the iodine in $\mathrm{PhICl}_{2}$ with either stoichiometric or excess pyridine or when dissolved in neat pyridine.

We then investigated the reaction between the stronger base 4-DMAP and $\mathrm{PhICl}_{2}$, which has also been reported to activate $\mathrm{PhICl}_{2}$ albeit with less efficacy than the catalytic reactions 
described as pyridine. ${ }^{5} \mathrm{PhICl}_{2}$ was dissolved in $\mathrm{CD}_{3} \mathrm{CN}$ and a solution of 4-DMAP was added. An aliquot was immediately removed for NMR spectroscopy. Protonated 4-DMAP, PhI and one other species containing 4-DMAP were identified. N-hexane was added to precipitate the protonated 4-DMAP. The solvent was removed and the residue redissolved in $\mathrm{CDCl}_{3}$. $\mathrm{Phl}$ was still present and a 4-DMAP containing species contained a singlet, and 2 doublets in the aryl region integrating with a 1:1:1 ratio, and one methyl resonance integrating to 6 -protons. This pattern is consistent with substitution of a proton with another atom on the aryl ring. Addition of $\mathrm{HOTf}$ resulted in precipitation of a white solid. Redissolving the solid in $\mathrm{CH}_{3} \mathrm{CN}$ for mass spectrometry analysis gave a strong signal at $\mathrm{m} / \mathrm{z}=157$, with an isotope pattern consistent with one chlorine. Single crystals were grown from a $\mathrm{CH}_{3} \mathrm{CN}$ solution via vapour diffusion of $\mathrm{Et}_{2} \mathrm{O}$ and $\mathrm{X}$-ray diffraction analysis gave the pyridinium triflate salt of $\mathbf{3}$ (Scheme 4, Figure 3), which is consistent with the activation of the ring towards EAS by the amino group, and does not displace chloride from $\mathrm{PhICl}_{2}$ to give $[\mathrm{PhICl}(4-\mathrm{DMAP})]^{+}$or a 4-coordinate complex analogous to 2 . Use of 2 equivalents of 4-DMAP gives complete conversion. These results indicate that 4-DMAP also cannot activate $\mathrm{PhICl}_{2}$ as proposed, as half of the catalytic amount of 4-DMAP would be converted into 3 and the other half sequestered as the hydrochloride salt.

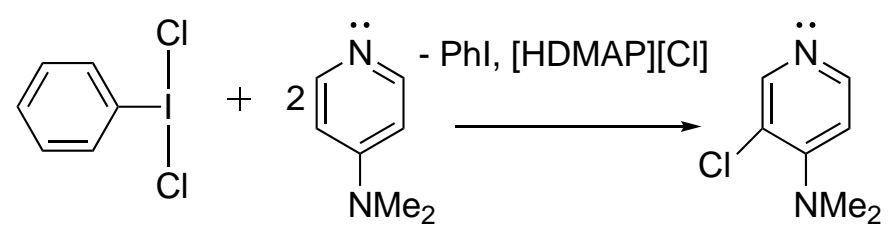

Scheme 4. Reaction of $\mathrm{PhICl}_{2}$ with 4-DMAP. 


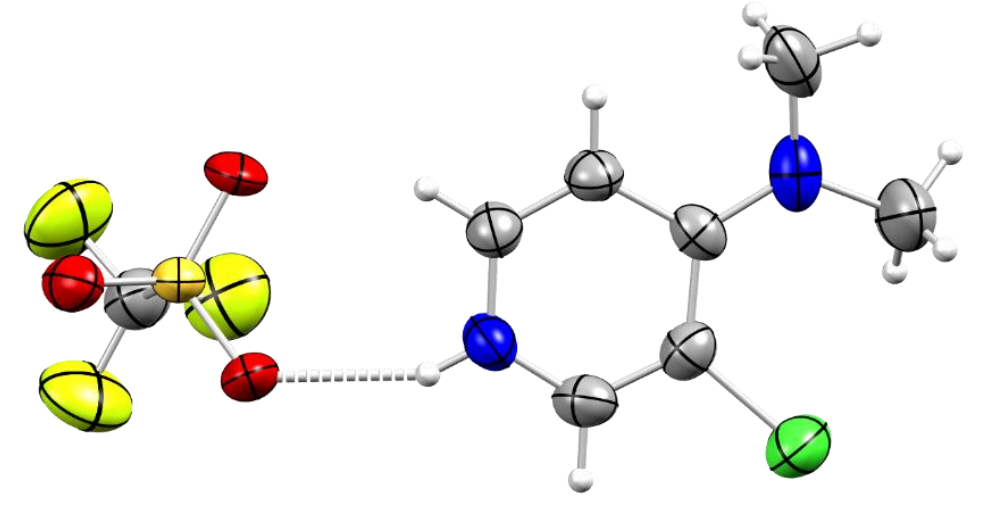

Figure 3. ORTEP representation of $\mathbf{3}$ shown with $70 \%$ probability ellipsoids.

The interaction of $\mathrm{PhICl}_{2}$ and $\mathbf{2}$ were then investigated through DFT calculations. The optimized geometry for 2 (B3LYP-D3(BJ)/def2-TZVPPD with acetonitrile solvation) gives a slightly elongated I-N bond and slightly shortened C-I bond at 2.856 and $2.118 \AA$, respectively, as compared to the experimental results. The calculated $\Delta G$ for the addition of pyridine to $\mathrm{PhICl}_{2}$ is $+12.1 \mathrm{~kJ} / \mathrm{mol}$ at the B3LYP-D3(BJ)/def2-QZVPPD(SMD, acetonitrile)//B3LYPD3(BJ)/def2-TZVPPD(SMD, acetonitrile) level of theory. With other methods (DSDPBEP86, $\omega B 97 X D$, DLPNO-CCSD $(T)$ ), solvents (dichloromethane, pyridine), and employing a quasiharmonic model the calculated $\Delta G$ remains consistently positive in sign but small in magnitude with values in the range of +9.8 to $+18.2 \mathrm{~kJ} / \mathrm{mol}$. The small positive $\Delta G$ is consistent with experimental observations with crystals of $\mathbf{2}$ only produced in neat pyridine that drives equilibrium towards formation of $\mathbf{2}$, with low temperature for crystallization reducing the effect of entropy, and is also consistent with the binding constant of $0.7 \mathrm{M}^{-1}$ from the NMR titration experiments between $\mathrm{PhICl}_{2}$ and pyridine

The displacement of chloride by pyridine to give $[\mathrm{Phl}(\mathrm{Pyr}) \mathrm{Cl}]^{+}$as previous proposed is thermodynamically unfavourable, with $\Delta G$ of $+30.0 \mathrm{~kJ} / \mathrm{mol}$. With dichloromethane or pyridine 
solvent models $\Delta G$ is +70.3 and $52.5 \mathrm{~kJ} / \mathrm{mol}$, respectively. At all levels of theory considered the calculated $\Delta G$ for displacement of chloride is much more unfavourable than for coordination of pyridine to $\mathrm{PhICl}_{2}$ to form compound 2 .

We also considered alternative pathways to produce the hypothesized $[\mathrm{Phl}(\mathrm{Pyr}) \mathrm{Cl}]^{+}$ intermediate. Loss of $\mathrm{Cl}^{-}$from $\mathrm{PhICl}_{2}$ is unfavourable by $94.9 \mathrm{~kJ} / \mathrm{mol}(\Delta G)$, while loss of $\mathrm{Cl}^{-}$from 2 to yield $[\mathrm{Phl}(\mathrm{Pyr}) \mathrm{Cl}]^{+}$is unfavourable by $17.9 \mathrm{~kJ} / \mathrm{mol}$.

In combination with the results from the synthetic studies described above it is concluded that displacement of a chloride by pyridine is not the mechanism by which $\mathrm{PhICl}_{2}$ is activated. It is possible that complex $\mathbf{2}$ is the activated species, so we examined the calculated properties of the pyridine adduct and compared them to free $\mathrm{PhICl}_{2}$.

Calculated CM5 and NPA atomic charges (B3LYP-D3(BJ)/def2-TZVPPD(SMD, acetonitrile)) indicate that the atomic charges on $\mathrm{C}_{\mathrm{Ph}}$ (bonded to I), I, and $\mathrm{Cl}$, vary by less than $0.04 \mathrm{e}$ between 2 and $\mathrm{PhICl}_{2}$ (Figure 3). The optimised $\mathrm{I}-\mathrm{C}$ and $\mathrm{I}-\mathrm{Cl}$ bond distances are also similar in $\mathrm{PhICl}_{2}$ and 2, with $\mathrm{I}-\mathrm{C}$ bond distances of 2.107 and $2.118 \AA$, and $\mathrm{I}-\mathrm{Cl}$ bond distances of 2.543 and $2.549 \AA$, respectively. The similar atomic charges and bond distances indicate that the electronic environment in $\mathrm{PhICl}_{2}$ is not significantly impacted by coordination of pyridine in $\mathbf{2}$. 

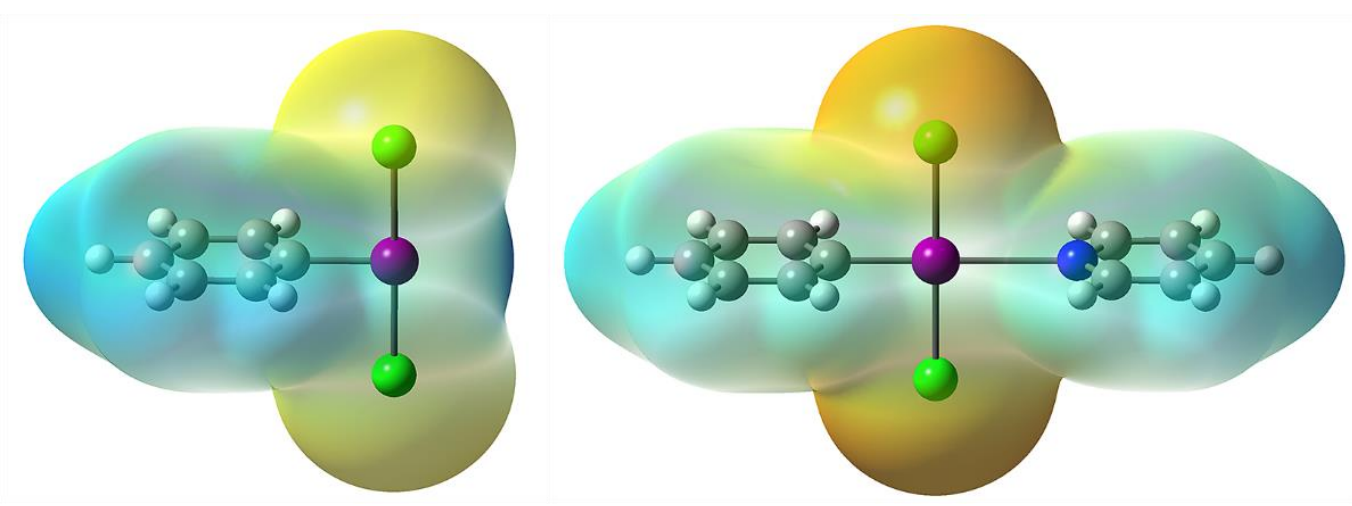

Figure 3. Plots of electrostatic potential (ESP) of (a) $\mathrm{PhICl}_{2}$ and (b) $\mathrm{PhICl}_{2}-\mathrm{Pyr}$ (compound 2). B3LYP-D3(BJ)/def2-TZVPPD(SMD, acetonitrile) results, isosurface from -6E-2 (dark yellow) to $+6 \mathrm{E}-2$ (blue).

It is possible that pyridine might also transiently interact with chlorine along the $\mathrm{I}-\mathrm{Cl}$ bond axis, however, attempts at optimizing a geometry from a starting point with the pyridine interacting with a chlorine atom resulted in the pyridine dissociating.

The lowest unoccupied $\mathrm{MO}$ (LUMO) for $\mathrm{PhICl}_{2}$ is a sigma-symmetric antibonding orbital orientated along the $\mathrm{Cl}-\mathrm{I}-\mathrm{Cl}$ bond axes (Figure 4). The LUMO+1 is found $1.46 \mathrm{eV}$ higher in energy than the LUMO and is also a sigma-symmetric antibonding orbital orientated along the I-C bond axis, which is the orbital the lone pair of the pyridine attacks, despite being higher in energy than the $\mathrm{Cl}-\mathrm{I}-\mathrm{Cl}$ based LUMO.
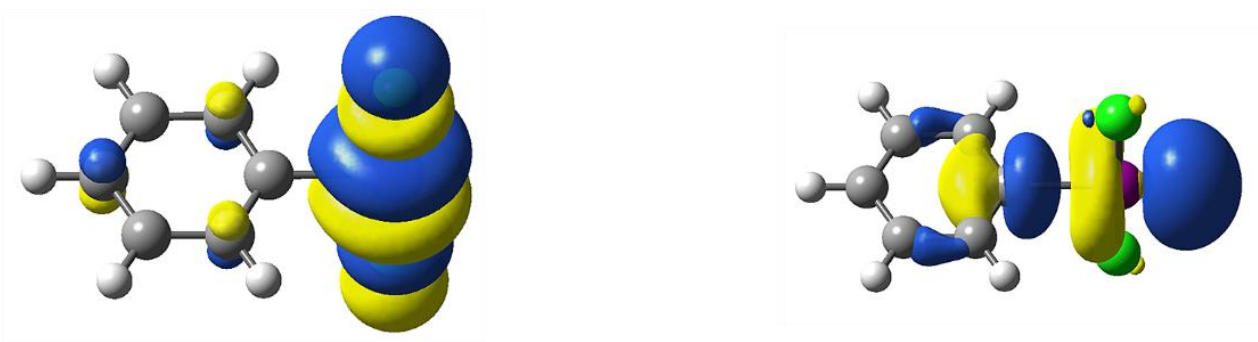

Figure 4. Plots of the LUMO (left) and LUMO +1 (right) for $\mathrm{PhICl}_{2}$. 
The calculated electrostatic potential of $\mathrm{PhICl}_{2}$ in Figure 3 clearly shows a more positive sigma hole at the iodine atom than is found at the chlorines, despite the $\mathrm{Cl}-\mathrm{I}-\mathrm{Cl}$ accepting orbital being lower in energy, and consistent with charge density studies described above.

In the pyridine adduct (2) the LUMO remains a sigma symmetric antibonding orbital orientated along the $\mathrm{Cl}-\mathrm{I}-\mathrm{Cl}$ bond axis. Relative to $\mathrm{PhICl}_{2}$ the orbital is $0.1 \mathrm{eV}$ higher in energy, which indicates that the adduct should be a poorer electron acceptor than free $\mathrm{PhICl}_{2}$ and a less active source of electrophilic chlorine.

\section{CONCLUSIONS}

We have shown here that pyridine does not displace chloride from $\mathrm{PhICl}_{2}$ and thus a $[\mathrm{Phl}(\mathrm{Pyr})(\mathrm{Cl})]^{+}$cation is likely not the reactive intermediate in the chemistry described by Murphy and co-workers. Pyridine clearly has an activating role of some kind based on their observations, what this is remains an open question. For $\mathrm{PhICl}_{2}$ the calculated $\mathrm{LUMO}$ and the experimentally determined and calculated sigma hole are not aligned, and the nucleophile (pyridine) in this case preferentially interacts with the sigma hole aligned with the LUMO+1, rather than the more accessible sigma symmetric LUMO.

\section{Acknowledgements}

We thank La Trobe University, The University of Queensland and the Australian Research Council (FT16010007, LE170100144, DP190102036 and DP200100013) for support. We thank $\mathrm{NCl}$, Intersect, and La Trobe University for generous allocations of computing resources. 


\section{REFERENCES}

1. Sousa e Silva, F. C.; Tierno, A. F.; Wengryniuk, S. E. Hypervalent iodine reagents in high valent transition metal chemistry. Molecules 2017, 22, 780-834.

2. Yoshimura, A.; Zhdankin, V. V. Advances in synthetic applications of hypervalent iodine compounds. Chem. Rev. 2016, 116, 3328-3435.

3. Zhdankin, V. V.; Stang, P. J. Chemistry of polyvalent iodine. Chem. Rev. 2008, 108, 5299-5358.

4. Tao, J.; Tran, R.; Murphy, G. K. Dihaloiodoarenes: Dihalogenation of phenylacetate derivatives. J. Am. Chem. Soc. 2013, 135, 16312-16315.

5. Murphy, G. K.; Abbas, F. Z.; Poulton, A. V. Dichlorination of oxindole derivatives using (Dichloroiodo)-benzene. Adv. Synth. Catal. 2014, 356, 2919-2923.

6. Hepples, C.; Murphy, G. K. Synthesis of 3,3-dichloro-2-oxindoles from isatin-3-ptosylhydrazones and (dichloroiodo)benzene. Tett. Lett. 2015, 56, 4971-4974.

7. Coffey, K. E.; Moreira, R.; Abbas, F. Z.; Murphy, G. K. Synthesis of 3,3-dichloroindolin2-ones from isatin-3-hydrazones and (dichloroiodo)benzene. Org. Biomol. Chem. 2015, 13, 682-685.

8. Zhao, Z.; Kulkarni, K. G.; Murphy, G. K. Synthesis of aryldihalomethanes by denitrogenative dihalogenation of benzaldehyde hydrazones. Adv. Synth. Catal. 2017, 359, 2222-2228.

9. Montanari, V.; DesMarteau, D. D.; Pennington, W. T. Synthesis and structure of novel perfluorinated iodinanes. Journal of Molecular Structure 2000, 550-551, 337-348 DOI: https://doi.org/10.1016/S0022-2860(00)00502-0.

10. Hansen, N. K.; Coppens, P. Testing aspherical atom refinements on small-molecule data sets. Acta Crystallographica Section A 1978, 34 (6), 909-921 DOI: doi:10.1107/S0567739478001886.

11. Clark, T.; Hennemann, M.; Murray, J. S.; Politzer, P. Halogen Bonding: The SigmaHole. J. Mol. Model. 2007, 13, 291-294.

12. Puttreddy, R.; Jurcek, O.; Bhowmik, S.; Makela, T.; Rissanen, K. Very strong -N-X+-O$\mathrm{N}+$ halogen bonds. Chem. Commun. 2016, 52, 2338-2341.

13. Setter, C. J.; Whittaker, J. J.; Brock, A. J.; Athukorala Arachchige, K. S.; McMurtrie, J. C.; Clegg, J. K.; Pfrunder, M. C. Straightening out halogen bonds. CrystEngComm 2020, 22 (10), 1687-1690 DOI: 10.1039/d0ce00176g.

14. Weiss, R.; Seubert, J. Electrostatic activation of hypervalent organo-iodine compounds: Bis(onio)-substituted aryliodine(III) salts. Angew. Chem. Int. Ed. 1994, 33, 891893.

15. Pell, T. P.; Couchman, S. A.; Ibrahim, S.; Wilson, D. J. D.; Smith, B. J.; Barnard, P. J.; Dutton, J. L. Diverse reactions of PhI(OTf)2 with common 2-electron ligands: Complex formantion, oxidation and oxidative coupling. Inorg. Chem. 2012, 51, 13034-13040. 16. Shaw, A. J. M.; Corbo, R.; Wilson, D. J. D.; Dutton, J. L. A 2,2-bipyridine coordination complex of [ICl2]+. Dalton Trans. 2015, 44, 15083-15087.

17. Hokamp, T.; Mollari, L.; Wilkins, L. C.; Melen, R. L.; Wirth, T. Alternative Strategies with iodine: fast access to previously inaccesible iodine(III) compounds. Angew. Chem. Int. Ed. 2018, 57, 8306-8309.

18. Thordarson, P. Determining association constants from titration experiments in supramolecular chemistry. Chem. Soc. Rev. 2011, 40, 1305-1323. 
19. http://supramolecular.org/ TOC Fig 